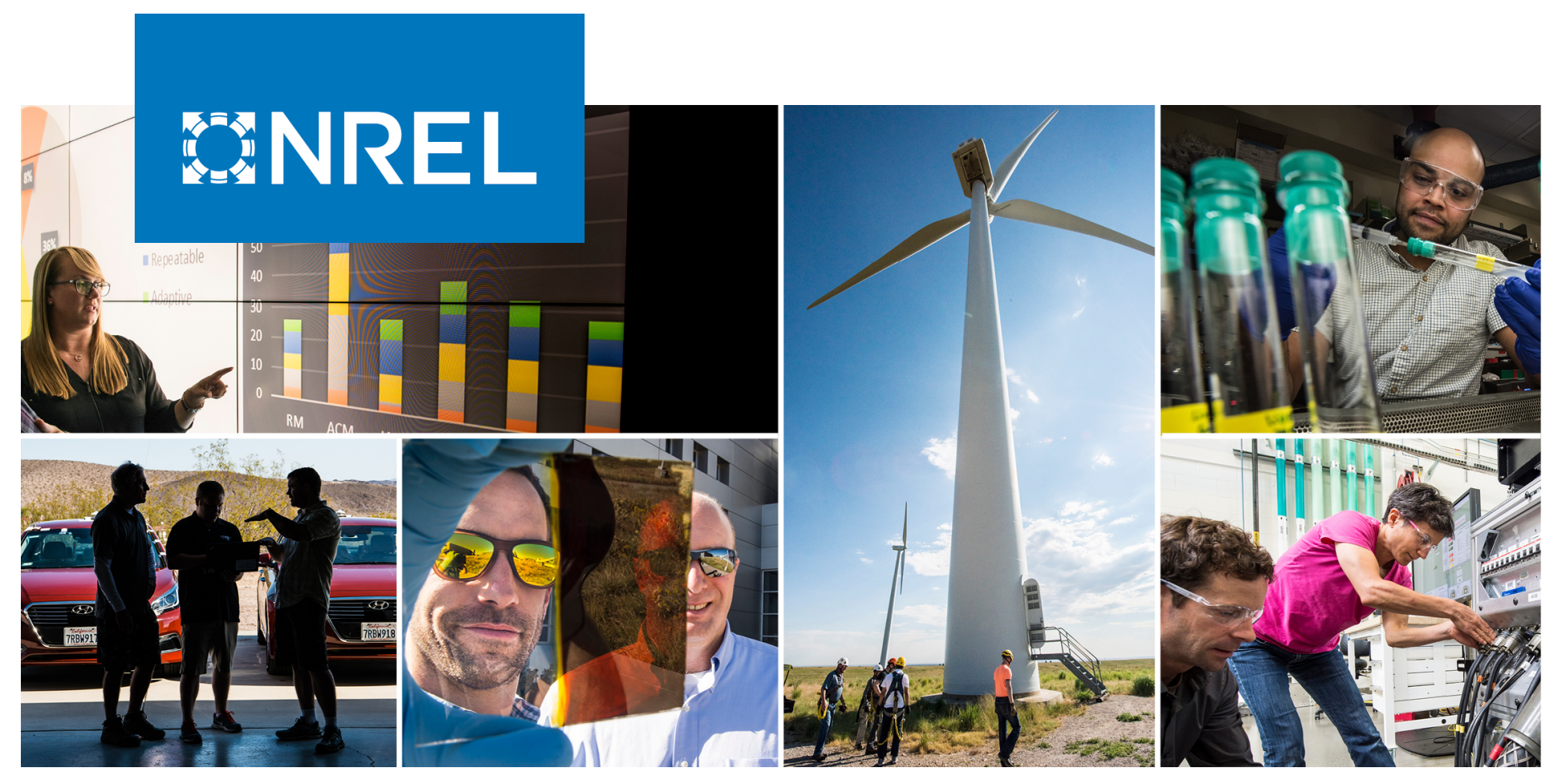

\title{
Distributed Minimization of the Power Generation Cost in Prosumer-Based Distribution Networks
}

\section{Preprint}

Guido Cavraro, ${ }^{1}$ Andrey Bernstein, ${ }^{1}$ Ruggero Carli, ${ }^{2}$ and Sandro Zampieri²

${ }_{2}^{1}$ National Renewable Energy Laboratory
2 University of Padova, Italy

Presented at the 2020 American Control Conference (ACC) July 1-3, 2020

NREL is a national laboratory of the U.S. Department of Energy Office of Energy Efficiency \& Renewable Energy

Operated by the Alliance for Sustainable Energy, LLC

This report is available at no cost from the National Renewable Energy Laboratory (NREL) at www.nrel.gov/publications.
Conference Paper

NREL/CP-5D00-76367

September 2020 


\title{
GNREL
}

\section{Distributed Minimization of the Power Generation Cost in Prosumer-Based Distribution Networks}

\section{Preprint}

\author{
Guido Cavraro, ${ }^{1}$ Andrey Bernstein, ${ }^{1}$ Ruggero Carli, ${ }^{2}$ \\ and Sandro Zampieri ${ }^{2}$ \\ ${ }^{1}$ National Renewable Energy Laboratory \\ 2 University of Padova, Italy
}

\section{Suggested Citation}

Cavraro, Guido, Andrey Bernstein, Ruggero Carli, and Sandro Zampieri. 2020. Distributed Minimization of the Power Generation Cost in Prosumer-Based Distribution Networks: Preprint. Golden, CO: National Renewable Energy Laboratory. NREL/CP-5D00-76367. https://www.nrel.gov/docs/fy20osti/76367.pdf.

NREL is a national laboratory of the U.S. Department of Energy Office of Energy Efficiency \& Renewable Energy Operated by the Alliance for Sustainable Energy, LLC

This report is available at no cost from the National Renewable Energy Laboratory (NREL) at www.nrel.gov/publications.

Contract No. DE-AC36-08GO28308
Conference Paper

NREL/CP-5D00-76367

September 2020

National Renewable Energy Laboratory 15013 Denver West Parkway Golden, CO 80401 303-275-3000 • www.nrel.gov 


\section{NOTICE}

This work was authored in part by the National Renewable Energy Laboratory, operated by Alliance for Sustainable Energy, LLC, for the U.S. Department of Energy (DOE) under Contract No. DE-AC36-08GO28308. Funding provided by U.S. Department of Energy and supported in part by the Laboratory Directed Research and Development (LDRD) Program at NREL. The views expressed herein do not necessarily represent the views of the DOE or the U.S. Government.

This report is available at no cost from the National Renewable Energy Laboratory (NREL) at www.nrel.gov/publications.

U.S. Department of Energy (DOE) reports produced after 1991 and a growing number of pre-1991 documents are available free via www.OSTI.gov.

Cover Photos by Dennis Schroeder: (clockwise, left to right) NREL 51934, NREL 45897, NREL 42160, NREL 45891, NREL 48097, NREL 46526.

NREL prints on paper that contains recycled content. 


\title{
Distributed Minimization of the Power Generation Cost in Prosumer-Based Distribution Networks
}

\author{
Guido Cavraro, Andrey Bernstein, Ruggero Carli, and Sandro Zampieri
}

\begin{abstract}
Traditionally, electrical power was generated in big power plants. The cost of producing energy was related to the cost of fuel, e.g., carbon or gas, and by the cost of maintaining the power plants. With the advent of distributed energy resources, power can be produced directly at the edge of the electrical network by a new type of agents: the prosumers. Prosumers are entities that both consume and generate power, e.g., by means of photovoltaic panels. The cost of the power produced by prosumers is no longer related to fuel consumption since energy coming from distributed generators is essentially free. Rather, the cost is related to the remuneration that is due to the prosumers for the services they provide. The proposed control strategy minimizes the active power generation cost in the aforementioned scenario. The control scheme requires that the prosumers measure their voltage and then adjust the amount of injected power, according to a continuous time feedback control law that is indeed a projected gradient descent strategy. Simulations are provided in order to illustrate the algorithm behavior.
\end{abstract}

\section{INTRODUCTION}

The advent of distributed energy resources (DERs), like wind turbines, photovoltaic panels, or other renewable energy sources, is going to dramatically change the actual electrical grid [1] as a massive number of small power generators will be deployed in the distribution grid. On one hand, a number of improvements for the electrical system will be achieved with the integration of the distributed energy resources in the distribution network, e.g. voltage profile improvements, reduction of line loss and power generation cost and other ancillary services [2]. On the other hand, distribution networks can suffer instabilities or damages if the (possibly) millions hosted DERs do not coordinate their power injections. This motivates the recent interest in developing algorithms that solve optimal power flow (OPF) problems for distribution networks, while in the past they were focused only on transmission networks. Utilities have

G. Cavraro and A. Bernstein are with the Power Systems Engineering Center, National Renewable Energy Laboratory (NREL), Golden, USA. Email: name.surname@nrel.gov.

R. Carli and S. Zampieri are with the Dept. of Information Engineering, University of Padova, Italy. Email: \{carlirug, zampi\}@dei.unipd.it.

This work was authored in part by the National Renewable Energy Laboratory (NREL), operated by Alliance for Sustainable Energy, LLC, for the U.S. Department of Energy (DOE) under Contract DE-AC3608GO28308 and supported in part by the Laboratory Directed Research and Development (LDRD) Program at NREL. The views expressed in the paper do not necessarily represent the views of the DOE or the U.S. Government. The U.S. Government retains and the publisher, by accepting the paper for publication, acknowledges that the U.S. Government retains a nonexclusive, paid-up, irrevocable, worldwide license to publish or reproduce the published form of this work, or allow others to do so, for U.S. Government purposes. to solve optimal power flow (OPF) problems to find a power system operating point that minimizes a cost function, e.g., the generation cost or the line losses, satisfying the power demand and some operative constraints, like limitation on the generation capability or voltage magnitude limits.

OPF problems for transmission networks are usually tackled by centralized off-line algorithms which (i) collect all the necessary field data, (ii) compute the optimal configuration, and (iii) dispatch the power production to the generators. Similar off-line solution strategy has been applied to distribution networks too, e.g., algorithms based on the alternating direction method of multipliers (ADMM) has been developed [3]. They require a large number of iterations and a high computational burden to converge, mainly due to the nonlinear relations among powers and voltages which make the OPF problem non-convex. To overcome these drawbacks, the OPF problem has been reformulated as a rank-constrained semidefinite program conveniently convexified by dropping the rank constraint [4]. Heed that in all the aforementioned solutions, the OPF solution is applied at the end of the optimization process, that requires either a global knowledge of the system state or a number of communication rounds necessary for the solution numerical computation.

This approach is not practical in distribution networks, because of the power demand fast variability and in the generators' generation capabilities, and to the fact that generators can connect or disconnect, requiring an automatic reconfiguration of the grid control infrastructure (the so called "plug and play" approach). This explains the recent interest in online distributed feedback optimization algorithms, where the feedback is exploited to infer from local measurements, e.g., voltages or power injections, global information [5]-[7].

The algorithm proposed herein is (i) a continuous time control algorithm, that exploit as feedback variables the phasorial voltages and information about the power flowing through the substation (also termed PCC in the following), (ii) is inherently an online algorithm, and (iii) can be implemented in a distributed way. The OPF problem we consider aims at minimizing the global power generation cost. In our setup we consider two types of cost functions: one associated with the power produced by conventional power plants and coming from the external transmission network, and one associated with the DERs dispersed in the distribution network. The problem is tackled via a projected gradientbased approach that is shown to be provably convergent to an approximated optimal solution of the OPF problem. The mathematical proofs of the resulted presented next can be found in the paper extended version [8]. 


\section{NOTATION AND MATHEMATICAL PRELIMINARIES}

Upper- (lower-) case boldface letters denote matrices (column vectors). Sets are denoted with calligraphic symbols. Symbol ${ }^{\top}$ stands for transposition. Vectors 1 and $\mathbf{e}_{m}$ represent the all-one vectors and the $m$-th canonical vector, respectively. Given a set $\mathcal{A},|\mathcal{A}|$ is the number of elements in $\mathcal{A}$. The projection of a vector $\mathrm{x}_{0}$ onto a convex set $\mathcal{X}$ is denoted as $\Pi_{\mathcal{X}}\left(\mathbf{x}_{0}\right)=\arg \min _{\mathbf{x} \in \mathcal{X}}\left\|\mathbf{x}-\mathbf{x}_{0}\right\|$.

A convex cone $\mathcal{K}$ is a set such that, for every $\mathbf{x}_{1}, \mathbf{x}_{2} \in \mathcal{K}$, $a \mathbf{x}_{1} \in \mathcal{K}, a \geq 0$, and $a \mathbf{x}_{1}+(1-a) \mathbf{x}_{2} \in \mathcal{K}, 0 \leq a \leq 1$. Given a convex cone $\mathcal{K} \subset \mathbf{R}^{N}$, its polar cone $\mathcal{K}^{*}$ is the set $\mathcal{K}^{*}=\left\{\mathbf{y}: \mathbf{y}^{\top} \mathbf{x} \leq 0, \mathbf{x} \in \mathcal{K}\right\}$.

Lemma 1 (Lemma 1, [7]): Consider a convex cone $\mathcal{K} \subset$ $\mathbf{R}^{N}$ and a vector $\mathbf{a} \in \mathbf{R}^{N}$. Let $\mathbf{b}=\Pi_{\mathcal{K}}(\mathbf{a})$. Then, it holds $\mathbf{a}-\mathbf{b} \in \mathcal{K}^{*}$.

The tangent cone of a non-empty convex set $\mathcal{X}$ at $\mathrm{x} \in \mathcal{X}$ is the convex cone

$$
\mathcal{T}_{\mathcal{X}}^{\mathbf{x}}=\operatorname{cl}\left\{\mathbf{d} \in \mathbf{R}^{N}: \exists \epsilon>0, \mathbf{x}+\epsilon \mathbf{d} \in \mathcal{X}\right\} .
$$

The normal cone of a non-empty convex set $\mathcal{X}$ at $\mathrm{x} \in \mathcal{X}$ is the convex cone

$$
\mathcal{N}_{\mathcal{X}}^{\mathbf{x}}=\left\{\mathbf{d} \in \mathbf{R}^{N}: \mathbf{d}^{\top}(\mathbf{y}-\mathbf{x}) \leq 0, \forall \mathbf{y} \in \mathcal{X}\right\} .
$$

The tangent and the normal cone of $\mathcal{X}$ at $\mathrm{x}$ are such that $\mathcal{N}_{\mathcal{X}}^{\mathbf{X}}=\left(\mathcal{T}_{\mathcal{X}}^{\mathbf{X}}\right)^{*}[9]$.

\section{CYBER-PHYSICAL MODEL OF A DISTRIBUTION GRID}

A smart power distribution network can be described as a cyber-physical system in which

- The physical layer comprises the distribution infrastructure: lines, loads, microgenerators, and the substation;

- The cyber layer consists of intelligent agents provided with actuation, communication, sensing, and computational capabilities.

The physical layer can be modeled using an undirected graph $\mathcal{G}=(\mathcal{V}, \mathcal{E})$; the set $\mathcal{V}$, with cardinality $|\mathcal{V}|:=n+1$, collects nodes that are associated with electrical buses. The substation, also termed point of common coupling (PCC), is labeled as 0 . As a consequence, we can write $\mathcal{V}=$ $\{0,1, \ldots, n\}$. The set $\mathcal{E}, \mathcal{E} \subseteq \mathcal{V} \times \mathcal{V}$, with cardinality $|\mathcal{E}|:=m$, collects the edges that are associated with the electrical lines. Usually, distribution networks feature a radial topology; thus, $m=n$. Define the following quantities:

- $\mathbf{u} \in \mathbb{C}^{n}$, with $u_{v}$ being the voltage at bus $v$;

- $\mathbf{i} \in \mathbb{C}^{n}$, where $i_{v}$ is the current injected at node $v$;

- $\mathbf{s}=\mathbf{p}+i \mathbf{q} \in \mathbb{C}^{n}$, where $s_{v}, p_{v}$ and $q_{v}$ are the complex, the active and the reactive power injected at node $v$. Active and reactive powers will take positive values, i.e., $p_{v}, q_{v} \geq 0$ when they are injected in the grid by bus $v$. Conversely, $p_{v}, q_{v} \leq 0$ means that bus $v$ is absorbing power from the grid.

- $\mathbf{z}=\mathbf{r}+i \mathbf{x} \in \mathbb{C}^{m}$, where $z_{\ell}, r_{\ell}$ and $x_{\ell}$ are the impedance, the resistance and the reactance of line $\ell$.

The system state is described by the following system of equations:

$$
\left[\begin{array}{c}
i_{0} \\
\mathbf{i}
\end{array}\right]=\mathbf{Y}\left[\begin{array}{c}
u_{0} \\
\mathbf{u}
\end{array}\right]
$$

$$
\begin{aligned}
& u_{0}=U_{N} \\
& u_{v} \bar{i}_{v}=p_{v}+i q_{v} \quad v \neq 0 .
\end{aligned}
$$

Equation (1a), where $\mathbf{Y}$ is the bus admittance matrix, provides the relation between voltages and currents. Equation (1b) holds since the substation is modeled as an ideal voltage generator imposing the nominal voltage $u_{0}=U_{N}$. Finally, equation (1c) comes from the fact that all the nodes, except the PCC, are modeled as constant power buses. It can be shown that there exists a unique matrix $\mathbf{Z} \in \mathbb{C}^{n \times n}$, called the Green matrix, that allows us to write voltages as a function of currents via:

$$
\mathbf{u}=\mathbf{Z i}+\mathbf{1} U_{N} .
$$

Notably, the $\mathbf{Z}$ is symmetric and positive semidefinite (see [10]). Furthermore, it has been shown that the non-linear relation between power injections and voltages satisfies [10]

$$
\mathbf{u}=\frac{(\mathbf{R}+i \mathbf{X})}{U_{N}}(\mathbf{p}-i \mathbf{q})+\mathbf{1} U_{N}+o\left(\frac{1}{U_{N}}\right)
$$

where the little-o notation means that $\lim _{U_{N} \rightarrow \infty} \frac{o\left(f\left(U_{N}\right)\right)}{f\left(U_{N}\right)}=$ 0 . As a consequence, the voltage-powers relation can be approximated with the linear function

$$
\mathbf{u}=\frac{(\mathbf{R}+i \mathbf{X})}{U_{N}}(\mathbf{p}-i \mathbf{q})+\mathbf{1} U_{n}
$$

where the matrices $\mathbf{R}$ and $\mathbf{X}$ are the real and the imaginary part of $\mathbf{Z}$, respectively. Power loss equals the sum of the powers injected by the substation and all the prosumers; thus, $p_{0}$ can be written as

$$
p_{0}(\mathbf{p})=-\sum_{v=1}^{n} p_{v}+\ell(\mathbf{p})=-\mathbf{1}^{\top} \mathbf{p}+\ell(\mathbf{p}) .
$$

where $\ell(\mathbf{p})$ denotes the active power loss. The dependence of power loss from $\mathbf{q}$ is dropped, since in this paper the reactive power injections $\mathbf{q}$ are considered as fixed, non-controllable variables. The linearization (3) can be used to find convex approximations of the active power network loss

$$
\ell(\mathbf{p})=\mathbf{p}^{\top} \frac{\mathbf{R}}{U_{N}^{2}} \mathbf{p}+\mathbf{q}^{\top} \frac{\mathbf{X}}{U_{N}^{2}} \mathbf{q} .
$$

and of the power flowing through the substation

$$
p_{0}(\mathbf{p})=-\mathbf{1}^{\top} \mathbf{p}+\mathbf{p}^{\top} \frac{\mathbf{R}}{U_{N}^{2}} \mathbf{p}+\mathbf{q}^{\top} \frac{\mathbf{X}}{U_{N}^{2}} \mathbf{q} .
$$

We assume that every bus in the power network is a prosumer [11], except the PCC. A prosumer is a bus able to generate the power $p_{v}^{g} \geq 0$ ( $v$ is a producer) but with a power demand $p_{v}^{d}, q_{v} \leq 0$ too. That is, a prosumer $v$ is both a producer and a consumer of energy. Define, $\bar{p}_{v}:=p_{v}^{d}+p_{v}^{g}$, i.e., $\bar{p}_{v}$ is the sum between the power demand and the generation capability of prosumer $v$. We have two cases:

- When $\bar{p}_{v} \geq 0$, the generation capability of $v$ exceeds the power demand and $v$ behaves like a generator. Its power output $p_{v}$ is controllable and satisfies $0 \leq p_{v} \leq \bar{p}_{v}$;

- When $\bar{p}_{v} \leq 0$, the power demand exceeds the generation capability of $v$ and $v$ behaves like an uncontrollable load requiring $p_{v}=\bar{p}_{v}$. 


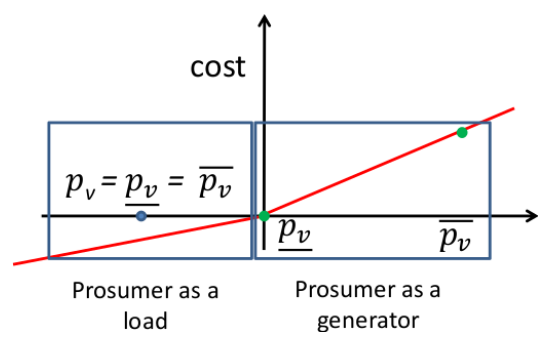

Fig. 1. Power cost function associated with a prosumer.

Each prosumer (referred to also as agent) is provided with sensing capability in the form of a phasor measurement unit (PMU). Finally, we assume that the power grid has a Network Supervisor (NS) able to measure the amount of power flowing into the distribution grid through the PCC. The NS acts as an intermediary between the prosumers and the distribution system operator, receiving updates on the generation cost of $p_{0}$, and broadcasting to all the prosumers information that will be used in the control algorithm.

\section{PROBLEM FORMULATION}

Next, a distributed feedback control algorithm will be devised to minimize the power generation cost. The generation cost is often chosen to be a polynomial, e.g., a quadratic function of the type [4], [12], [13]

$$
f_{v}\left(p_{v}\right)=\left\{\begin{array}{ll}
\alpha_{v, 2} p_{v}^{2}+\alpha_{v, 1} p_{v}+\alpha_{v, 0} & p_{v} \geq 0 \\
0 & p_{v}<0
\end{array} .\right.
$$

This is a common choice in the literature that well describe the case in which power is produced in power plants; however, it does not capture the case in which power is produced by a DER, like a photovoltaic panel. In the latter case, energy is produced at zero cost; rather, the power cost is the remuneration due to the generator owners for the power injection. In summary,

- When a prosumer is behaving like a generator, the remuneration is proportional to the quantity of energy injected into the grid, with a proportionality constant that depends on the contractual agreement with the utility;

- When a prosumer is behaving like a load, it will not receive a remuneration; instead, the prosumer has to pay the power consumed, whose price is proportional to the power absorbed. As a result, the utility must sustain a negative price, i.e. it earns money.

The model we use for the remuneration (see Figure 1) comes straightforwardly from the previous reasoning, and it is

$$
\hat{f}_{v}\left(p_{v}\right)=\left\{\begin{array}{ll}
c_{v, G} p_{v} & p_{v} \geq 0 \\
c_{v, L} p_{v} & p_{v}<0
\end{array} \quad \forall v \in \mathcal{V}\right.
$$

In this framework there are mainly two possible scenarios. The first one is related to the "prosumers point of view", in which the prosumers compete in order to maximize the profit they earn from selling power to the grid. Overall, this boils down to a game among the agents. A first treatment of this scenario can be found in [14].

The second scenario is instead related to the "utility point of view", where the total cost accounts for the production cost of the energy injected by the PCC (that comes from big generation plants such as nuclear or hydroelectrical plants) and for the remuneration to be paid to the owners of DRES. In this framework, the goal of the utility is to minimize the total cost while satisfying some operative constraints.

In this paper we focus on the second scenario. To have a lighter notation, we assume that all the agents are paid in the same way, that is,

$$
c_{v, G}=c_{G} \quad \forall v \in \mathcal{V} \backslash\{0\}
$$

and we model $f_{0}\left(p_{0}\right)$ as

$$
f_{0}\left(p_{0}\right)= \begin{cases}c_{0} p_{0} & p_{0} \geq 0 \\ 0 & p_{0}<0\end{cases}
$$

However, our results can be easily generalized to the case in which (9) does not hold and the power generation cost at the PCC is modeled with classic cost functions, e.g., (7). We make also the following assumption.

Assumption 2: The reward $c_{G}$ is positive, that is $c_{G}>0$.

Formally, the problem we are interested into can be stated as the following optimization problem

$$
\begin{array}{ll}
\min _{\mathbf{p}} & \sum_{v=1}^{n} f_{v}\left(p_{v}\right)+f_{0}\left(p_{0}\right) \\
\text { s. t. } & p_{0}(\mathbf{p})=-\mathbf{1}^{\top} \mathbf{p}+\mathbf{p}^{\top} \frac{\mathbf{R}}{U_{N}^{2}} \mathbf{p}+\mathbf{q}^{\top} \frac{\mathbf{X}}{U_{N}^{2}} \mathbf{q} \\
& \mathbf{p} \in \mathcal{B}
\end{array}
$$

where

- equation (11b) models active power conservation;

- $\mathcal{B}$ is the feasible set, that is

$$
\mathcal{B}=\left\{\mathbf{p}: \underline{p}_{v} \leq p_{v} \leq \bar{p}_{v}\right\}
$$

If prosumer $v$ behaves as a load, then $\underline{p}_{v}=\bar{p}_{v}<0$. If prosumer $v$ behaves as a generator, then $\underline{p}_{v}=0$ and $0<\bar{p}_{v}<\infty$. Thus, the set $\mathcal{B}$ is bounded.

- the cost functions $f_{v}\left(p_{v}\right)$ 's are defined as

$$
f_{v}\left(p_{v}\right):=c_{G} p_{v} .
$$

The function $f_{v}\left(p_{v}\right)$ is different from the piece-wise actual cost $\hat{f}_{v}\left(p_{v}\right): f_{v}\left(p_{v}\right)$ is linear. Nonetheless, the minimizer of (11) is the same as if the $\hat{f}_{v}\left(p_{v}\right)$ 's would have been used, even though the minimum is different. In fact, $f_{v}\left(p_{v}\right)=\hat{f}_{v}\left(p_{v}\right)$ for $p_{v} \geq 0$, i.e., where $p_{v}$ is an actual optimization variable; while $f_{v}\left(p_{v}\right) \neq \hat{f}_{v}\left(p_{v}\right)$ for $p_{v}<0$, where constraint (11c) forces $p_{v}=\underline{p}_{v}=\bar{p}_{v}$.

Define $f(\mathbf{p})=\sum_{v=1}^{n} f_{v}\left(p_{v}\right)+f_{0}\left(p_{0}\right)$. Thanks to (10) and (12), we can write

$$
f(\mathbf{p})= \begin{cases}f^{+}(\mathbf{p}) & \text { if } \mathbf{p} \in \mathcal{S}^{+} \cup \mathcal{S}^{0} \\ f^{-}(\mathbf{p}) & \text { if } \mathbf{p} \in \mathcal{S}^{-} \cup \mathcal{S}^{0}\end{cases}
$$


where

$$
\begin{aligned}
& f^{+}(\mathbf{p})=\left(c_{G}-c_{0}\right) \mathbf{1}^{T} \mathbf{p}+c_{0}\left(\mathbf{p}^{\top} \frac{\mathbf{R}}{U_{N}^{2}} \mathbf{p}+\mathbf{q}^{\top} \frac{\mathbf{X}}{U_{N}^{2}} \mathbf{q}\right) \\
& f^{-}(\mathbf{p})=c_{G} \mathbf{1}^{T} \mathbf{p} \\
& \mathcal{S}^{+}=\left\{\mathbf{p}: p_{0}(\mathbf{p})>0\right\} \quad \mathcal{S}^{-}=\left\{\mathbf{p}: p_{0}(\mathbf{p})<0\right\} \\
& \mathcal{S}^{0}=\left\{\mathbf{p}: p_{0}(\mathbf{p})=0\right\} .
\end{aligned}
$$

$\mathcal{S}^{0}$ is a variety separating $\mathcal{S}^{+}$from $\mathcal{S}^{-}$. The cost $f(\mathbf{p})$ is a continuous function: $f^{+}(\mathbf{p})$ and $f^{-}(\mathbf{p})$ are continuous in $\mathcal{S}^{+} \cup \mathcal{S}^{0}$ and in $\mathcal{S}^{-} \cup \mathcal{S}^{0}$, respectively; moreover, $f^{+}(\mathbf{p})=$ $f^{-}(\mathbf{p})$ for all $\mathbf{p} \in \mathcal{S}^{0}$. Furthermore, $f(\mathbf{p})$ is differentiable in $\mathcal{S}^{+}$and in $\mathcal{S}^{-}$, but not in $\mathcal{S}^{0}$. The solution of problem 11 is characterized by the following proposition, proved in [6].

Proposition 3: Consider problem (11), let Assumption 2 hold and denote with $\mathbf{p}^{*}$ an optimal solution of (11). Then, every optimal configuration $\mathbf{p}^{*}$ is such that $p_{0}\left(\mathbf{p}^{*}\right) \geq 0$, i.e., it belongs to $\mathcal{S}^{+} \cup \mathcal{S}^{0}$.

\section{A Continuous-Time Algorithm}

Problem (11) could be solved by a continuous-time projected gradient descent of the cost function. However, remind that Proposition 3 ensures that the optimum configuration lies in $\mathcal{S}^{+} \cup \mathcal{S}^{0}$. To speed up the rate of convergence, a reasonable strategy is to steer the prosumers power injections $\mathbf{p}$ in the region $\mathcal{S}^{+} \cup \mathcal{S}^{0}$ as fast as possible when $\mathbf{p} \in \mathcal{S}^{-}$, instead of executing the mere gradient descent on $f$. Hence, we propose the following strategy:

- if $p_{0}(\mathbf{p})<0$, agents aims at increasing $p_{0}$ following the rule

$$
\dot{\mathbf{p}}(t)=\Pi_{\mathcal{T}_{\mathcal{B}}^{\mathbf{p}(t)}}\left(\nabla p_{0}(\mathbf{p}(t))\right)
$$

- if $p_{0}(\mathbf{p}) \geq 0$, agents perform the cost function gradient descent.

$$
\dot{\mathbf{p}}(t)=\Pi_{\mathcal{T}_{\mathcal{B}}^{\mathbf{p}(t)}}\left(-\nabla f^{+}(\mathbf{p}(t))\right)
$$

The overall control rule can be written as

$$
\dot{\mathbf{p}}(t)=\Pi_{\mathcal{T}_{\mathcal{B}}^{\mathbf{p}(t)}}(\varphi(\mathbf{p}(t)))
$$

where

$$
\varphi(\mathbf{p})= \begin{cases}-\nabla f^{+}(\mathbf{p})=\left(c_{0}-c_{G}\right) \mathbf{1}-\frac{c_{0}}{U_{N}^{2}} \mathbf{R p} & \mathbf{p} \in \mathcal{S}^{+} \\ \nabla p_{0}(\mathbf{p})=-\mathbf{1}+\frac{1}{U_{N}^{2}} \mathbf{R} \mathbf{p} & \mathbf{p} \in \mathcal{S}^{-}\end{cases}
$$

Heed that the vector field (17) is continuous on the sets $\mathcal{S}^{+}$and $\mathcal{S}^{-}$, but it is discontinuous and not defined on $\mathcal{S}^{0}$, since $f$ is not differentiable in $\mathcal{S}^{0}$. A widely accepted way to extend (17) on $\mathcal{S}^{0}$ is given by the Filippov convex method, which considers the following set-valued extension, or convexification, of $\varphi(\mathbf{p})$ [15]. Precisely, let $\varphi^{0}(\mathbf{p})$ be the extension of $\nabla f(\mathbf{p})$ on $\mathcal{S}^{0}$. According to the Filippov method,

$$
\varphi^{0}(\mathbf{p}) \in \overline{c o}\left\{\nabla p_{0}(\mathbf{p}),-\nabla f^{+}(\mathbf{p})\right\}
$$

where $\overline{c o}\left\{\nabla p_{0}(\mathbf{p}),-\nabla f^{+}(\mathbf{p})\right\}$ denotes the smallest convex set that contains $\nabla p_{0}(\mathbf{p})$ and $-\nabla f^{+}(\mathbf{p})$. Hence, for $\mathbf{p} \in \mathcal{S}^{0}$, we can write

$$
\varphi^{0}(\mathbf{p})=-\alpha(\mathbf{p}) \nabla f^{+}(\mathbf{p})+(1-\alpha(\mathbf{p})) \nabla p_{0}(\mathbf{p})
$$

where $\alpha(\mathbf{p}))$ is a suitable non-negative variable, $0 \leq \alpha(\mathbf{p}) \leq$ 1 , which can take different values leading to:

- Attractive sliding mode. When both $-\nabla f^{+}(\mathbf{p})$ and $\nabla p_{0}(\mathbf{p})$ are pointing towards $\mathcal{S}^{0}$, or

$$
-\nabla f^{+}(\mathbf{p})^{\top} \nabla p_{0}(\mathbf{p})<0
$$

an attractive sliding mode occurs. When (20) is satisfied, $\mathbf{p}$ will move along $\mathcal{S}^{0}$ giving rise to a sliding motion. During the sliding motion, $\alpha(\mathbf{p})$ is such that $\varphi^{0}(\mathbf{p})$ is tangent to $\mathcal{S}^{0}$, or $\varphi^{0}(\mathbf{p})^{\top} \nabla p_{0}(\mathbf{p})=0$. It can be easily shown that

$$
\alpha(\mathbf{p})=\frac{\left\|\nabla p_{0}(\mathbf{p})\right\|^{2}}{\nabla p_{0}(\mathbf{p})^{\top}\left(\nabla f^{+}(\mathbf{p})+\nabla p_{0}(\mathbf{p})\right)}
$$

and that $\alpha(\mathbf{p})>0$.

- Transversal intersection. When $\nabla f^{+}(\mathbf{p})$ and $\nabla p_{0}(\mathbf{p})$ have the same orientation, that is

$$
-\nabla f^{+}(\mathbf{p})^{\top} \nabla p_{o}(\mathbf{p})>0
$$

there is no sliding motion and the trajectory of $\mathbf{p}(\cdot)$ will leave the surface $\mathcal{S}^{0}$. The parameter $\alpha(\mathbf{p})$ is chosen as $\alpha(\mathbf{p})=1$.

Based on the previous considerations, the gradient field (17) can be extended as

$$
\varphi(\mathbf{p})= \begin{cases}-\nabla f^{+}(\mathbf{p}) & \mathbf{p} \in \mathcal{S}^{+} \\ \nabla p_{0}(\mathbf{p}) & \mathbf{p} \in \mathcal{S}^{-} \\ \varphi^{0}(\mathbf{p}) & \mathbf{p} \in \mathcal{S}^{0}\end{cases}
$$

Beside the discontinuity of $\varphi(\mathbf{p})$ in $\mathcal{S}^{0}$, the dynamic (16) may be discontinuous also on the boundaries of the feasible set $\mathcal{B}$, where the gradient is projected on the cone of the feasible directions. For these reasons, the standard notion of solution for ordinary differential equations does not apply. In this paper, the trajectory $\mathbf{p}:[0, t] \rightarrow \mathbb{R}^{n}$ is a Caratheodory solution on the interval $[0, t]$ if it is absolutely continuous and satisfies (16) almost everywhere in $[0, t]$. The system considered in this paper guarantees the uniqueness of the Caratheodory solution, see [16].

The next Proposition, which is the main result of this paper, shows that the dynamic (16) is asymptotically stable and that steers the system towards the solution of (11).

Proposition 4: Consider problem (11) and the dynamic (16). Let Assumption 2 hold. Then, (11) has a unique solution $\mathbf{p}^{*}$ and the trajectory $t \rightarrow \mathbf{p}(t)$ is asymptotically stable and converges to the unique optimum $\mathbf{p}^{*}$.

\section{A DISCRETE-TIME FEEDBACK CONTROL IMPLEMENTATION}

The control strategy (16) cannot be actually implemented, since inverters or power electronic devices in general perform control actions on discrete times. For this reason, we introduce the discrete-time version (16):

$$
\mathbf{p}(t+1)= \begin{cases}\Pi_{\mathcal{B}}\left(\mathbf{p}(t)-\epsilon_{+} \nabla f^{+}(\mathbf{p}(t))\right. & \text { if } p_{0}(\mathbf{p})>0 \\ \Pi_{\mathcal{B}}\left(\mathbf{p}(t)+\epsilon_{-} \nabla p_{0}(\mathbf{p}(t))\right. & \text { if } p_{0}(\mathbf{p})<0\end{cases}
$$


where $\epsilon_{+}, \epsilon_{-}$are a suitable positive constant. The continuous time dynamic (16) can be seen as the limit of the discrete time dynamic (24) when $\epsilon_{+}$and $\epsilon_{-}$tend to zero.

To avoid the computational burden of a centralized algorithm, we seek for a distributed implementation of (24), i.e., we aim at designing a control scheme that can be performed by each prosumer having just a local and partial (thus not global) knowledge of the grid state.

Note that the gradients $\nabla f^{+}(\mathbf{p}(t))$ and $\nabla p_{0}(\mathbf{p}(y))$ cannot be computed by each prosumer using only local information. Rather, global information, i.e., the whole power injection vector $\mathbf{p}$ and the grid structure $\mathbf{R}$, is needed, in contrast with our desire for designing a distributed algorithm. However, an estimate of $\mathbf{R p}$ can actually be computed locally. Assume that all the power lines in the grid have the same inductance/resistance ratio, but possibly different impedance magnitude, i.e., $z_{\ell}=e^{i \theta}\left|z_{\ell}\right|$ for any $\ell$ in $\mathcal{E}$ and for a fixed $\theta$. In this case, equation (3) becomes

$$
\mathbf{u}=e^{i \theta}|\mathbf{Z}|(\mathbf{p}-i \mathbf{q})+\mathbf{1} U_{N}
$$

where the matrix $|\mathbf{Z}|$ collects the absolute values of the entries of $\mathbf{Z}$. This assumption is satisfied when the inductance/resistance ratio of the power lines of the grid is relatively homogeneous, which is reasonable in many practical cases, e.g., see the IEEE standard testbeds [17] and [18, Table I]. Under (25), we can estimate $\mathbf{R p}$ as

$$
\mathbf{R} \mathbf{p}=\cos \theta \Re\left(e^{-i \theta}\left(\mathbf{u}-\mathbf{1} U_{N}\right)\right) .
$$

Equation 26 can be used finally to obtain the approximations

$$
\begin{aligned}
& \nabla f^{+}(\mathbf{p})=\left(c_{G}-c_{0}\right) \mathbf{1}+\frac{c_{0} \cos \theta}{U_{N}^{2}} \Re\left(e^{-i \theta}\left(\mathbf{u}-\mathbf{1} U_{N}\right)\right) \mathbf{1} \\
& \nabla p_{0}(\mathbf{p})=-\mathbf{1}+\frac{\cos \theta}{U_{N}^{2}} \Re\left(e^{-i \theta}\left(\mathbf{u}-\mathbf{1} U_{N}\right)\right) \mathbf{1}
\end{aligned}
$$

Note that every prosumer can compute its component of $\nabla f^{+}(\mathbf{p})$ knowing its own voltage phasor, the prices $c_{G}$ and $c_{0}$, and the parameter $\theta$, since

$$
\begin{aligned}
& {\left[\nabla f^{+}(\mathbf{p})\right]_{v}=\left(c_{G}-c_{0}\right)+\frac{c_{0} \cos \theta}{U_{N}^{2}} \Re\left(e^{-i \theta}\left(u_{v}-U_{N}\right)\right)} \\
& {\left[\nabla p_{0}(\mathbf{p})\right]_{v}=-1+\frac{\cos \theta}{U_{N}^{2}} \Re\left(e^{-i \theta}\left(u_{v}-U_{N}\right)\right) .}
\end{aligned}
$$

By plugging (27) and (28) into equation (24), we obtain the control rule performed by the prosumers. Trivially, the projection onto the feasible set can be done locally. To perform (24), besides local information, prosumers need to know the current power flowing the substation, the substation voltage, and the current price $c_{0}$. The NS is in charge of metering $p_{0}$ and $U_{N}$ and broadcasting their value and $c_{0}$ to the prosumers. Based on the above description, it is clear what is the feedback scheme that underlies the procedure we propose: during each iteration agents and the NS sense the grid, communicate, compute the power setpoint and then actuate it. The control strategy, formally described in Algorithm I, require only an estimate of the resistance/reactance ratio to be executed. Hence, it can be considered as a model free optimization algorithm.

\section{AlgORITHM I}

Let $\epsilon$ be a suitable positive constant. At the $(t+1)$-th cycle. The NS performs the following actions:

1. Measure the power $p_{0}(\mathbf{p}(t))$ and the voltage $U_{N}$.

2. Broadcast $p_{0}(\mathbf{p}(t)), U_{N}$ and $c_{0}$.

Prosumer $v$ performs the following actions

1. Measure its own voltage $\left.u_{v}(t)\right)$.

2. Receive the quantities $p_{0}(\mathbf{p}(t)), U_{N}$ and $c_{0}$.

3. Computes its gradient component through (27) or (28).

4. Computes, using equation (24), and applies the active power setpoint.

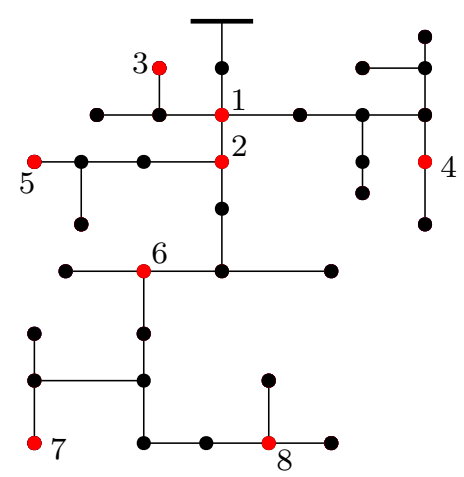

Fig. 2. Schematic representation of the IEEE 37-bus test feeder. Red nodes represent prosumers behaving as generators, as their power generation capability exceed their power requirement. Black nodes instead represent prosumer behaving as loads, whose power demand is bigger than their generation capabilities.

\section{Simulations}

The algorithm has been tested on a single-phase equivalent of the IEEE 37-bus test feeder, reported in Figure 2. The load buses are a mixture of constant-current, constant-impedance and constant-power loads, with a total active power power demand of almost $2 \mathrm{MW}$ and reactive power demand of almost 1 MVAR [17]. The line impedances differ from edge to edge, however, the inductance/resistance ratio exhibits a smaller variation, ranging from $\angle z_{\ell}=0.47$ to $\angle z_{\ell}=0.59$. Eight prosumers, able to generate more power than they require, behave as generators with controllable power output. Their maximum active power capabilities have been set to values that go from 85 to $490 \mathrm{~kW}$. The algorithm presented in Section VI was simulated on a nonlinear exact power flow solver [19]; on the same solver, the true solution of the optimization $\mathbf{p}^{*}$ was computed. The step-size $\epsilon$ was chosen small to make the trajectory similar to the continuous time version. To study the convergence of the feedback optimization algorithm, introduce the variable $d=\left\|\mathbf{p}-\mathbf{p}^{*}\right\|$.

Firstly, we simulate the case in which $c_{G}<c_{0}$, i.e., the case in which it is cheapest to import power from the external grid. Precisely, we normalized $c_{0}=1$ and 

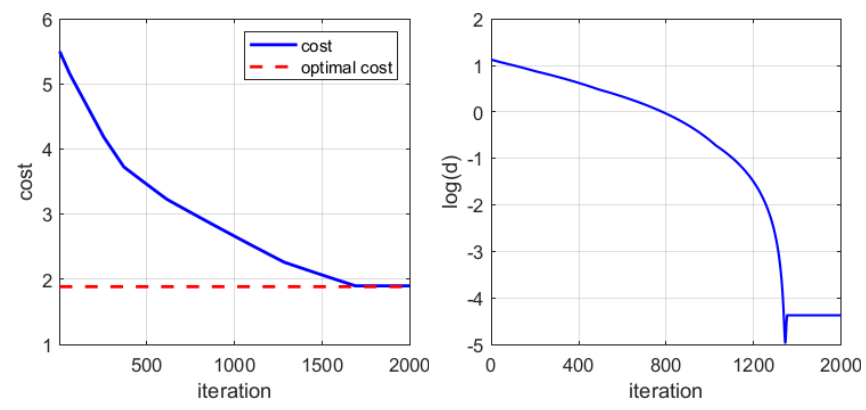

Fig. 3. An algorithm numerical simulation with $c_{G}=1.5$ and $c_{0}=1$.
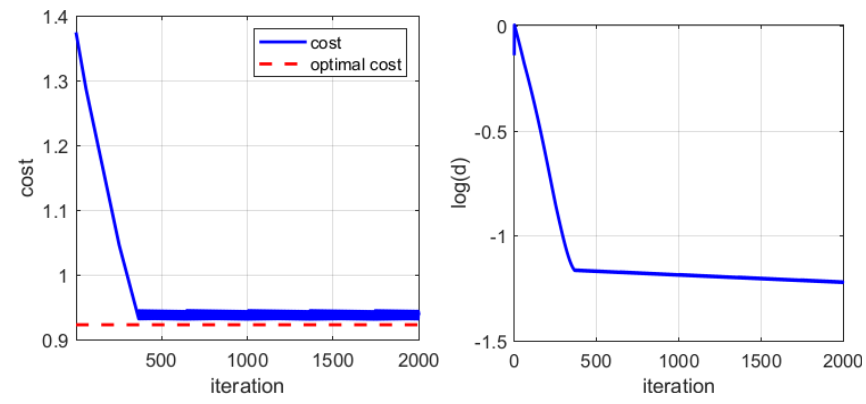

Fig. 4. An algorithm numerical simulation with $c_{G}=0.5$ and $c_{0}=1$.

set $c_{G}=1.5$. In this case, $\mathbf{p}^{*} \in \mathcal{S}^{+}$. The results are reported in Fig. 3. Secondly, the case where $c_{0}<c_{G}$, i.e., when buying energy from the distributed generators is more convenient, was inspected. Fig. 4 shows the results for $c_{0}=1, c_{G}=0.5$. In this case, $\mathbf{p}^{*} \in \mathcal{S}^{0}$ and a sliding motion appeared. Some comments are in order. First, the cost of the equilibria configurations is slightly higher than the cost of the true minimum, i.e., the equilibrium is suboptimum, see Fig. 3 and Fig. 4, left panels. This is because of the approximations used to distribute the algorithm. Second, in the case $c_{0}=1, c_{G}=0.5$, the reaching phase, i.e., the part of the trajectories in which $\mathbf{p}$ reaches the surface $\mathcal{S}^{0}$, lasts about 400 iterations. Once p starts sliding on the surface $\mathcal{S}^{0}$, the trajectory of $\mathbf{p}$ slows down, see Fig. 3, right panel. Third, when $c_{0}=1, c_{G}=0.5$, and $\mathbf{p}$ is on the sliding surface, the trajectory experienced the typical sliding mode chattering, which appears because the control strategy is implemented in discrete time rather than in continuous time, and it is evident on the trajectory of $p_{0}$, see Fig. 5.

\section{CONCLUSION}

This paper presents and analyzes a feedback control algorithm for the minimization of the generation cost in a distribution network. The main features of our approach are the following. Firstly, it considers the case in which the cost of the power is not related to actual generation cost, but rather to economical agreements between utilities and prosumers. Secondly, it can be referred to as a "quasi local" approach. Each prosumer is able to implement the control rule by using only local information and some common knowledge that it is broadcasted by the NS. Finally, simulations on a standard testbed prove the effectiveness of the proposed

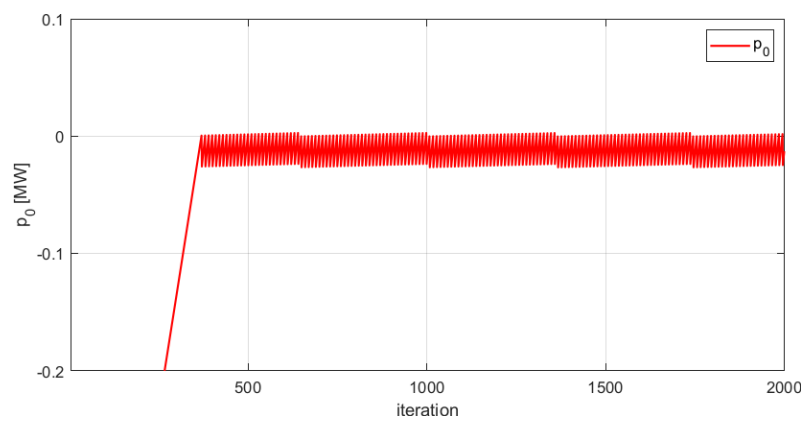

Fig. 5. Trajectory of $p_{0}(\mathbf{p})$ in the same numerical simulation depicted in Figure 4.

control scheme.

\section{REFERENCES}

[1] A. Ipakchi and F. Albuyeh, "Grid of the future," Power and Energy Magazine, IEEE, vol. 7, no. 2, pp. 52-62, 2009.

[2] F. Katiraei and M. Iravani, "Power management strategies for a microgrid with multiple distributed generation units," Power Systems, IEEE Transactions on, vol. 21, no. 4, pp. 1821-1831, 2006.

[3] T. Erseghe, "Distributed optimal power flow using admm," IEEE Trans. on Power Systems, vol. 29, no. 5, pp. 2370-2380, Sep. 2014.

[4] J. Lavaei and S. H. Low, "Zero duality gap in optimal power flow problem," IEEE Transactions on Power Systems, vol. 27, no. 1, pp. 92-107, Feb 2012.

[5] G. Cavraro and R. Carli, "Local and distributed voltage control algorithms in distribution networks," IEEE Transactions on Power Systems, vol. 33, no. 2, pp. 1420-1430, March 2018.

[6] G. Cavraro, R. Carli, and S. Zampieri, "A distributed control algorithm for the minimization of the power generation cost in smart micro-grid," in Conference on Decision and Control (CDC14), 2014.

[7] C. Chang, M. Colombino, J. Corté, and E. Dall'Anese, "Saddle-flow dynamics for distributed feedback-based optimization," IEEE Control Systems Letters, vol. 3, no. 4, pp. 948-953, Oct 2019.

[8] G. Cavraro, A. Bernstein, R. Carli, and S. Zampieri, "Distributed minimization of the power generation cost in prosumer-based distribution networks," 2020.

[9] R. T. Rockafellar and R. J.-B. Wets, Variational analysis. Springer Science \& Business Media, 2009, vol. 317.

[10] S. Bolognani and S. Zampieri, "A Distributed Control Strategy for Reactive Power Compensation in Smart Microgrids," IEEE Transactions on Automatic Control, vol. 58, no. 11, pp. 2818-2833, 2013.

[11] S. Grijalva and M. U. Tariq, "Prosumer-based smart grid architecture enables a flat, sustainable electricity industry," in Innovative Smart Grid Technologies (ISGT), 2011 IEEE PES. IEEE, 2011, pp. 1-6.

[12] E. Devane and I. Lestas, "Stability and convergence of distributed algorithms for the opf problem," in 52nd IEEE Conference on Decision and Control, 2013.

[13] E. Mallada and A. Tang, "Dynamics-aware optimal power flow," in 52nd IEEE Conference on Decision and Control, 2013.

[14] G. Cavraro and L. Badia, "A game theory framework for active power injection management with voltage boundary in smart grids," in European Control Conference ECC 2013, 2013.

[15] L. Dieci and L. Lopez, "Sliding motion in filippov differential systems: theoretical results and a computational approach," SIAM Journal on Numerical Analysis, vol. 47, no. 3, pp. 2023-2051, 2009.

[16] J. Cortes, "Discontinuous dynamical systems," IEEE Control Systems Magazine, vol. 28, no. 3, pp. 36-73, June 2008.

[17] W. H. Kersting, "Radial distribution test feeders," in Power Engineering Society Winter Meeting, 2001. IEEE, vol. 2. IEEE, 2001, pp. 908-912.

[18] G. Cavraro, V. Kekatos, and S. Veeramachaneni, "Voltage analytics for power distribution network topology verification," IEEE Trans. Smart Grid, vol. PP, no. 99, pp. 1-1, 2017.

[19] R. D. Zimmerman, C. E. Murillo-Sánchez, and R. J. Thomas, "MATPOWER: steady-state operations, planning and analysis tools for power systems research and education," IEEE Transactions on Power Systems, vol. 26, no. 1, pp. 12-19, Feb. 2011. 